\title{
Intermittent Prediction Method Based On Marcov Method And Grey Prediction Method
}

\author{
Aiping Jiang \\ Junjun Gao \\ Ying Wan \\ Xinyi Zhao \\ Siqi Shan
}

SHU-UTS SILC Business School, Shanghai University, Jiading District, Shanghai, China

\section{doi: 10.19044/esj.2016.v12n15p81 URL:http://dx.doi.org/10.19044/esj.2016.v12n15p81}

\begin{abstract}
This paper concentrates on the intermittent demand for electric power supply and studies the method of demand prediction. This chapter first divides the demand for electric power supply into two statistical sequences: (1) sequence of demand occurrence, among which " 1 "stands for the occurrence of demand, " 0 "means that the demand fails to occur; (2) sequence of demand quantity. Next the author predicts the moment of time and the number of times $n$ that demand occurs within a specific time interval in the future based on 0-1 sequence using Markov arrival process (MAP). Then the paper forecasts the demand quantity in subsequent $n$ intervals using Grey prediction model GM $(1,1)$ based on the sequence of demand quantity. Finally, the author places the demand quantity in the $\mathrm{n}$ intervals in order at the moments where demand occurs to get the predicted result of demand for electric material with intermittent demand. According to instance analysis, the integrated approach mentioned in this paper surpasses existing methods in providing accurate prediction on data of product with intermittent demand.
\end{abstract}

Keywords: Intermittent Demand; Demand Prediction; Grey Prediction Model

\section{Introduction}

Normal operation of electrical power system is based on the condition where electrical equipments are working in order. Accidental damages or malfunction of these equipments could lead to the breakdown of the whole electrical system. Having a quantity of electric power materials (equipment and spare parts) in store is essential to deal with these 
emergencies. The storage of a range of inventory facilitates instant fixture and equipment placement, thus ensures normal electric supply. Currently, analytic method dominates quantitative decision studies, in terms of spare parts management in a global context. In addition, the inventory level usually depends on the demand of maintenance points in a given period, but the variety of electrical materials leads to the low guarantee efficiency of the assumptions and simplification of analytic method. On one hand, certain kinds of materials are of large amount but low use rate. Storing these materials costs a great percentage of flow funds, which would impact the financial performance of the company. On the other hand, if no certain material is available when breakdown or fault happens, it is supposed to be ordered and produced instantly. Consequently, extra charges are required, which contributes to the rapid rise of maintenance cost, with far lower service level and continuously high cost level. The universal phenomenon of large backlog together with severe deficiency of electrical materials is a great bother to electric power companies. Therefore accurate predictions to scientifically and properly allocate material resources are significant to solve this problem. According to (Silver, 1981) and (Hua, Zhang, Yang, \& Tan, 2007), if the demand of most electrical materials are recorded once a week or month, or recorded according to the existence of interrupt, there are numbers of zero value which tends to present intermittent characteristics. Furthermore, the records of demand cannot be converted into a continuous one because converting the history data into continuous sequence makes it indeed useless for practices in real life. To be more specific, (Willemain, Smart, \& Schwarz, 2004) indicates that some electrical spare parts are merely ordered once in a couple of years, but their lead time are usually very short, from a few days to a couple of months. Thus, their annual demand records are of little importance for real-life practices. In other words, if existing continuous method were utilized to forecast the sporadic demand for such spare parts, the precision level would not be high. However, (Ghobbar \& Friend, 2003) and (Rustenburg, van Houtum, \& Zijm, 2001) note that the value of intermittent-demand electrical materials is rather high in real cases, thus the downtime caused by stockout of these expensive materials would bring about significantly high cost. Thereby, the demand prediction of highvalue materials is always the focus of most electrical corporations.

\section{Research Background Intermittent Demand Forecasting}

In current literature context, the main forecast methods for intermittent demand include exponential smoothing method, Croston method, Bootstrap method, fitting fractional step method and so forth. 
According to (Gardner, 1985), exponential smoothing method is wildly used to forecast continuous demand. Although it is not fit for intermittent demand forecast, Willemain et al., (2004) suggests that it can be improved for this purpose. For example, (Croston, 1972) comes up with Croston method, taking demand intervals (after how many intervals there is a demand) apart from demand size and using exponential smoothing method to calculate intervals and size separately. What should be noticed is that Croston is only suitable for spare parts whose lead time follows normal distribution. However, the lead time of different intermittent demand patterns actually does not obey normal distribution. Therefore, the Croston method is not accurate when applied to predict abnormally distributed demand. Moreover, although Croston is only capable of forecasting the average demand in every given time, there are still several limitations in terms of its intermittent demand forecast. Specifically, it can neither forecast the size of the demand, the time period when the demand occurs, nor the probabilities of the occurrence of estimated demand in every given time period, which is not consistent with the discontinuity of electrical materials.

As for Bootstrap, (Gamero, García, \& Reyes, 1998) describe that this method is a powerful estimation tool for sampling distributions and standard error. (Efron, 1979) originally introduced this method, Bootstrap, which generates virtual data from a sample of history records and forecasts the occurrence of demand with according probabilities and demand size. In the last step, what should be done is to repeat all the formal steps to generate many demand samples in order to get the distribution of demand in lead time. However, quite many drawbacks appear in the application of Bootstrap. First of all, the autocorrelation of real intermittent demands is not for sure. The more reasonable evaluation is that the accuracy of the forecast through Bootstrap cannot be guaranteed thus it is tough to insure the effectiveness of demand data that does not appear when demand involves more new random factors. Thirdly, the stability in this method may vary rapidly and difference between any two practices could never be zero, even very great (sometimes the best accuracy result could be 100 percent but the worst can be 0). In addition, (Snyder, 2002) indicates that Bootstrap may underestimate the requirement variation of lead times.

Apart from Bootstrap, fitting fractional step method (according to Leven and (Leven \& Segerstedt, 2004), normally to fit demand normal distribution, gamma distribution, Laplace distribution or Compound Poisson distribution are used), Bayesian method could cater for estimating intermittent demands as well. Fitting method is to extract actual statistical sequence from certain specific distribution and then to estimate future demands. (Grange, 1998) have pointed that using fitting method for intermittent demand is not precise and if the demand distribution could not 
be accurately predicted, this could contribute to severe results. Specifically speaking, overestimating demand rate or overestimating service level can both generate awful effects on firms. (Silver, 1968) takes use of Bayesian method to achieve intermittent demand estimation; the steps of it are as followings. Firstly, all need to be done is to get prior probability distribution according to the profitability of demand occurrence and then build posterior distribution on calculated posterior probability of lead times (the demand is multi-dimensional beta distribution). The next step is to confirm reorder point and order quantity under the precise that satisfying probability of storage. Nevertheless, the Bayesian method requires multi-sample and, in these samples, all possible demand quantities must appear, which might be difficult to be met for actual intermittent demand data.

In conclusion, in terms of intermittent statistical sequence, the predicting methods mentioned above request either large samples or the samples that could relatively strictly follow one certain type distribution, but most electrical materials cannot meet such requirements. As a result, this essay comes up with an integrated approach for intermittent demand on the basis of Marcov and Grey Prediction method. The first step is to divide the demand of electrical materials into two statistical sequences, namely "whether demand will occur" 0-1 sequence and demand size, when demand occurs, sequence. Secondly, Marcov arrival process (MAP) should be used to forecast time points where demands occur for 0-1sequence, followed by taking Grey Prediction to predict demand sizes. The whole procedure helps to formulate the final forecast demand result of electrical materials with discontinuity.

\section{Notation, Mathematical Description of the Integrated Forecasting Method}

In the first place, what needs to be done is to set the intermittent demand for electrical materials before $\mathrm{t}$ time point (includes $\mathrm{t}$ point), $X=\left[X_{1}, X_{2}, \cdots X_{t}\right]$, into two statistical sequences:

(1) whether demand will occur sequence, $T=\left[T_{1}, T_{2}, \cdots T_{t}\right]$, using " 1 " to express the occurrence of demand, " 0 " to present that demand does not occur;

(2) demand size sequence, $D=\left[D_{1}, D_{2}, \cdots D_{k}\right]$, namely the actual demand quantity when demand occurs; in next procedure, MAP is supposed to estimate $\mathrm{T}$ sequence, getting $T_{t+1}=T^{*}$; when $T^{*}=0$, there is no demand forecasted; when $T^{*}=1$, there will be a demand appear at $\mathrm{t}+1$ time point and 
it is time to use Grey Prediction method, GM $(1,1)$, if , $\hat{D}_{k+1}=D^{*}$, the final predicted value would be $\hat{X}_{k+1}=D^{*}$.

The processes above are shown as the picture below.

Fig.1. Flow chart of demand forecasting

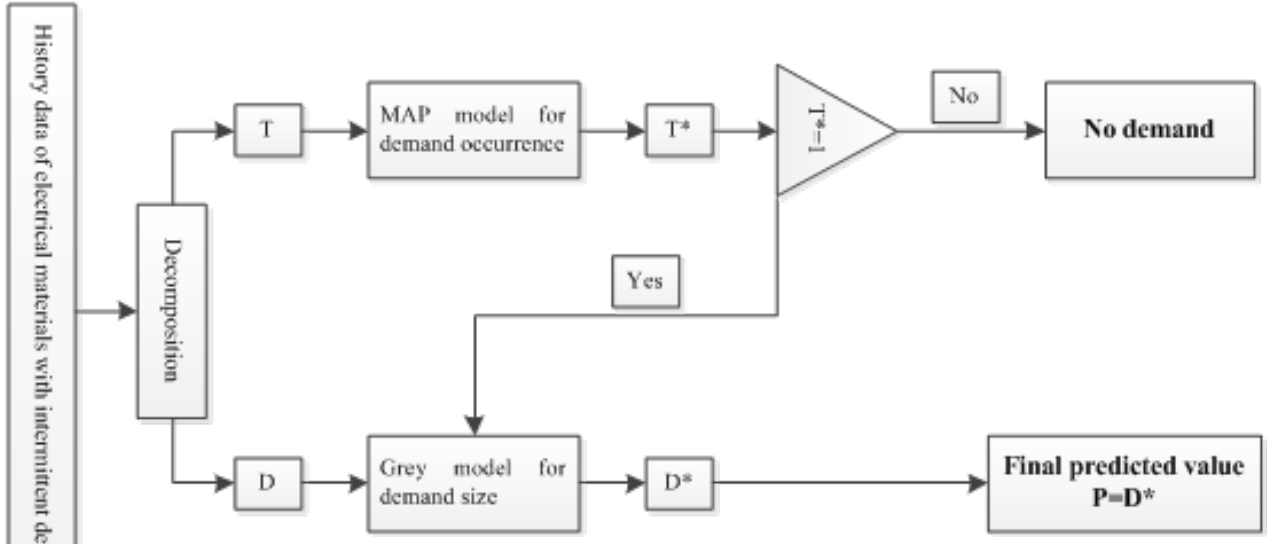

\section{Empirical Investigation}

\section{Model Simulation}

\section{Prediction of Demand Occurrence by MAP}

This paper takes use of the MAP to forecast demand of electrical materials, namely selects appropriate Marcov transition matrix, which is to convert 0-1 sequence that represents whether demand will happen into probability form to judge information of future demands. If the probability of value 0 is larger than that of value 1 , it illustrates that there will be no demand in the forecasting period and there is no need to occupy demand forecast; if the probability of the arrival of value 1 is larger than value 0 , there will be demand occurring in forecasting period.

Because there are merely two sorts of sequences which demonstrate whether demand will occur, demand occurrence sequence, of intermittent demand, 1 or 0 appears meaning there is a or no demand happen separately. For Marcov of only two alternatives, its one-step transition probability matrix can be defined as:

$$
P=0 \begin{array}{rl}
0 & 1 \\
1 & {\left[\begin{array}{rl}
1-a & a \\
b & 1-b
\end{array}\right], 0<a, b<1}
\end{array}
$$

Set as demand in period $n(n=1,2, \ldots, k)$, the process always could be regarded as a homogeneous Marcov chain and the state space is $\mathrm{T}=\{0$, 1 1\}.Confirming demand sequence MAP is to ensure the $n$ times of transition 
probability of Marcov chain, $P_{i j}(n)$, and this step needs to use well-known Chapman-Kolmogorov equation, abbreviating it as C-K equation in the following context. The definition of this equation is for one homogeneous Marcov chain $\left\{X_{n}, n=0,1,2, \ldots\right\}$, for any $m, n \in T$,

$$
P_{i j}(m+n)=\sum_{k=1}^{+\infty} P_{i k}(m) P_{k j}(n), i, j=1,2,3 \ldots
$$

The state description is as follows: at a given time point $t$, the state is $X(t)=a_{i}$. From this point, the state experiences a shift of $\mathrm{m}+\mathrm{n}$ and the state changes to $a_{j}$, namely $X(t+m+n)=a_{j}$. As long as the value of $\mathrm{m}+\mathrm{n}$ is fixed, no matter what the value of $m, n$ is, after experiencing $m+n$ times of shift the state $X(t+m+n)=a_{j}$ can always be achieved. Then the matrix form of C-K can be expressed as

$$
\begin{gathered}
P(n)=P(1) P(n-1)=P P(n-1)=P^{k-1} P(1) P(n-k) \\
P(m+n)=P(m) P(n)
\end{gathered}
$$

In this way, taking use of $\mathrm{C}-\mathrm{K}$ equation could get the following recurrence relation

$$
P(n)=P(1) P(n-1)=P P(n-1)=P^{k-1} P(1) P(n-k)
$$

Referring to the relation above, we could get $P(n)=P^{n}$ Then we can conclude that the n-step matrix of transition probability of homogeneous Marcov chain is the $n$ power one-step transition probability matrix.

According to $\mathrm{C}-\mathrm{K}$ equation, we could know that $P(n)=P^{n}$. One-step transition probability matrix $\mathrm{P}$ has two different eigen values $\lambda_{1}=1, \lambda_{2}=1-a-b$, using aesthetic to present $\mathrm{P}$ as the similar matrix of diagonal matrix $\Lambda$, which can be shown as the following equation:

$$
\Lambda=\left(\begin{array}{cc}
\lambda_{1} & 0 \\
0 & \lambda_{2}
\end{array}\right)=\left(\begin{array}{cc}
1 & 0 \\
0 & 1-a-b
\end{array}\right)
$$

The feature vectors of $\lambda_{1}, \lambda_{2}$ are

$$
d_{1}=\left[\begin{array}{c}
1 / \sqrt{2} \\
-1 / \sqrt{2}
\end{array}\right], d_{1}=\left[\begin{array}{c}
\frac{a}{\sqrt{a^{2}+b^{2}}} \\
\frac{b}{\sqrt{a^{2}+b^{2}}}
\end{array}\right], H=\left[\begin{array}{cc}
1 / \sqrt{2} & \frac{a}{\sqrt{a^{2}+b^{2}}} \\
-1 / \sqrt{2} & \frac{b}{\sqrt{a^{2}+b^{2}}}
\end{array}\right]
$$

Then $P=H \Lambda H^{-1}$. Through this method, n power one-step transition probability matrix for demand arrival could be drawn

$$
P=\left(H \Lambda H^{-1}\right)^{n}=H \Lambda^{n} H^{-1}
$$




$$
=\frac{1}{a+b}\left[\begin{array}{ll}
b & a \\
b & a
\end{array}\right]+\frac{(1-a-b)^{n}}{a+b}\left[\begin{array}{cc}
a & -a \\
-b & b
\end{array}\right], n=1,2,3 \ldots
$$

In this way, the probability of the state $(0,1)$ drawn from $n$ power matrix of transition probability that experiences $n$ times of shift is $\left(P_{0}, P_{1}\right)$ :

$$
\left(P_{0}, P_{1}\right)=[P(0) P(1)] P^{n}
$$

Among the equation, $\mathrm{P}(0)$ stands for the probability of zero value in the original state, and $\mathrm{P}(1)$ presents the probability of value 1 in its original state.

\section{Forecasting demand size by GM $(1,1)$}

Grey system theory is come up with Deng Julong professor in 1982. It is a forecasting method to study highly unstable systems and it is a system between the white system and the black system. Grey system theory takes “ knowing part information and part of information are unknown” as a small sample, lack of information and uncertain system as study target, by means of extracting valuable data from information known to achieve accurate illustration and effective supervision for systematic operation and evolution laws. Classic Grey Prediction model is a GM $(1,1)$ model whose basis theory is to use accumulation to generate a series when a time sequence presents no obvious tendency and the rising trend of such series is capable of being used to build a model and make a forecast taking grey factors into account. The next step is to do inverse calculations using serial-down methods and then recover original time series to get forecast results.

Grey Prediction model GM $(1,1)$ is a dynamic change model that uses linear first-order differential equation to describe the series of grey system. The building process is as the following: For non-negative smooth history consumption data sequence: $X^{(0)}=\left(x^{(0)}(1), x^{(0)}(2), \ldots, x^{(0)}(n)\right)$, use the following accumulating method to do one calculation:

$$
x^{(1)}(1)=x^{(0)}(1), x^{(1)}(2)=x^{(1)}(1)+x^{(0)}(2), \ldots, x^{(1)}(n)=x^{(1)}(n-1)+x^{(0)}(n),
$$

namely: $x^{(1)}(k)=\sum_{i=1}^{k} x^{(0)}(k) \quad k=1,2, \ldots, n$ the result of accumulation data sequence is $X^{(1)}=\left(x^{(1)}(1), x^{(1)}(2), \ldots, x^{(1)}(n)\right)$. Assuming that $X^{(1)}$ obeys exponential change rule, then the winterization equation chosen is

$$
\frac{d X^{(1)}}{d t}+\lambda X^{(1)}=u
$$

and in the equation, $\lambda$ is called developing grey number, $\mathrm{u}$ is called internal control grey number. These two grey numbers can be received by least square method: 


$$
\left(\begin{array}{l}
\lambda \\
\mu
\end{array}\right)=\left(A^{T} A\right)^{-1} A^{T} Y
$$

and matrix $\mathrm{A}$ and matrix $\mathrm{Y}$ are expressed as:

$$
\begin{array}{r}
A=\left(\begin{array}{c}
-Z^{(1)}(2) 1 \\
-Z^{(1)}(3) 1 \\
. \\
. . \\
-Z^{(1)}(n) 1
\end{array}\right), \quad Y=\left(\begin{array}{c}
-X^{(0)}(2) \\
-X^{(0)}(3) \\
. . \\
-X^{(0)}(n)
\end{array}\right) \\
Z^{(1)}=\left(Z^{(1)}(2), Z^{(1)}(3), \ldots, Z^{(1)}(n)\right) \text { is the background value and it is }
\end{array}
$$
usually equal to the average of two consecutive values of $X$, namely $Z^{(1)}(k)=0.5\left(x^{(1)}(k-1)+x^{(1)}(k+1)\right), \quad k=2,3, \ldots, n$. So, for the winterization equation (10) whose original condition is $Z^{(1)}(k)=0.5\left(x^{(1)}(k-1)+x^{(1)}(k+1)\right), \quad k=2,3, \ldots, n$, the solution is:

$$
\hat{X}^{(1)}(k)=\left(x^{(0)}(1)-\frac{\mu}{\lambda}\right) e^{-\lambda(k-1)}+\frac{\mu}{\lambda}, \quad k=1,2, \ldots
$$

Actually, this is the predictor formula of $X^{(1)}$. Following such procedures, the predictor formula of $X^{(0)}$ is supposed to be:

$$
\hat{X}^{(0)}(k)=\hat{X}^{(1)}(k)-\hat{X}^{(1)}(k-1), k=2,3, \ldots
$$

When $\mathrm{k}$ is greater than $\mathrm{n}$, the estimated result is available; in contrast, when $\mathrm{k}$ is less than or equal to $\mathrm{n}$, the value of simulation of history consumption data, is available.

\section{Algorithm Flow}

According to the previous analysis, the predict approach to electrical materials with intermittent characteristics is supposed to go through the following main calculation steps:

(1) Divide the history data of relevant materials into demand size sequence D and whether demand will happen series T;

(2) Using MAP prediction model to forecast series $\mathrm{D}$ and $\mathrm{T}$ to receive predicted value that stands for whether demand will occur, $\mathrm{T}^{*}$

(3) On the basis of the estimate result of step (2) to construct demand size forecasting. If step (2) predicts that there will be no demand, the outcome would be demand size $\mathrm{Q}=0$; otherwise, if $\mathrm{Q}=\mathrm{D}^{*}$, the outcome would be $\mathrm{D}^{*}$. 


\section{Forecasting Accuracy Measures}

In experiment, approaches for predicting demand occurrence, that is MAP, this paper will take use of Percentage of Occurrence Error, POE, to calculate the wrong percentage. Setting ${ }_{d_{i}}$ to present actual value $z_{i}$,

for estimated value. The definition of POE is:

$$
\begin{aligned}
& \text { when }\left\{\begin{array}{c}
d_{i}=0 \text { and } z_{i}=0 \\
\text { or }, O E_{i}=0 ; \\
d_{i} \neq 0 \text { and } z_{i} \neq 0
\end{array}\right. \\
& \text { when }\left\{\begin{array}{c}
d_{i}=0 \text { and } z_{i} \neq 0 \\
\text { or } \quad \text { or } z_{i}=0 \\
d_{i} \neq 0 \text { and }
\end{array}, O E_{i}=1\right. \text {. } \\
& P O E=\frac{\sum_{i=1}^{n} O E_{i}}{n}
\end{aligned}
$$

Total Accuracy $=(1-P O E) * 100 \%$. At the same time, this essay calculates the accuracy and inaccuracy of forecasted results separately and the definitions are shown as the followings:

When $d_{i}=0$ and $z_{i}=0, " 0 " O E_{i}=0$

When $d_{i}=0$ and $z_{i} \neq 0, " 0 " O E_{i}=1$

When $d_{i} \neq 0$ and $z_{i} \neq 0$, "1" $O E_{i}=0$

When $d_{i} \neq 0$ and $z_{i}=0$," $1 " O E_{i}=1$

The accuracy of demand prediction when demand occurs

$$
\text { Accuracy"1" }=\left(1-\frac{\sum_{i=1}^{n} " 1 " O E_{i}}{n}\right) * 100 \%
$$

The accuracy of demand prediction when demand does not occur

$$
\text { Accuracy"0" }=\left(1-\frac{\sum_{i=1}^{n} " 0 " O E_{i}}{n}\right) * 100 \%
$$

In addition, this paper will calculate the differences in estimated and actual times of demand occurrence as Miss Demand. In particular, Miss Demand = estimated times of demand occurrence - actual times of demand occurrence.

This variable is quite helpful to design inventory size in the actual inventory management of electrical materials in terms of the number. 
Because intermittent includes large number of zero value, in order to avoid that the denominator is zero leading to incapability of calculation, this paper will not choose Mean Absolute Error, MAE, instead of Mean Absolute Percentage Error, MAPE, as accuracy indicator to compare MAE with intermittent demand forecasting approaches mentioned above--- Croston method, Trend-monthly method and ES method for it. The calculation formula is as the following:

$$
\begin{array}{r}
M A E=\frac{1}{n} \sum_{i=1}^{n}\left|f_{i}-y_{i}\right|=\frac{1}{n} \sum_{i=1}^{n}\left|c_{i}\right| \\
R M S E=\sigma=\sqrt{\frac{\sum d_{i}^{2}}{n}}, i=1,2,3 \ldots \\
\text { MASE }=\frac{\frac{1}{L} \sum_{j=1}^{L}\left|y_{n+j}-y_{n}(j)\right|}{\frac{1}{n+1} \sum_{t=2}^{n}\left|y_{t}-y_{t-1}\right|}
\end{array}
$$

Denote: $\mathrm{n}$ is the length of historical time series, $\mathrm{L}$ is the length of the predicted periods.

\section{Empirical Research}

All the data collected was randomly selected from the monthly demand historical data base of Shanghai Electrical Company, especially from its warehouses on Jiulong Road, Wenxiang Road and Libing Road, in the period 2007 to 2012. Within this period, data collected during 2007 to 2011 was used as demand historical data, and data collected within the year 2012 was used as tested parameter. Due to the limitat of paragraph, this article only represented experimental result of twenty representative electrical materials, to prove the validity of intermittent demand prediction method.

Table 1 Forecasted results of 20materials for demand occurrence

\begin{tabular}{ccccc}
\hline $\begin{array}{c}\text { Country } \\
\text { Net Code }\end{array}$ & $\begin{array}{c}\text { Total } \\
\text { Accuracy }\end{array}$ & $\begin{array}{c}\text { Accuracy } \\
1\end{array}$ & $\begin{array}{c}\text { Accuracy } \\
0\end{array}$ & $\begin{array}{c}\text { Miss } \\
\text { Demand }\end{array}$ \\
\hline 500031955 & 1.0000 & 1.0000 & 1.0000 & 0 \\
500067198 & 0.9091 & 1.0000 & 0.0991 & 1 \\
500037055 & 1.0000 & 1.0000 & 1.0000 & 0 \\
500026753 & 1.0000 & 1.0000 & 1.0000 & 0 \\
500026753 & 0.9091 & 0.9091 & 1.0000 & -1 \\
500065263 & 0.9091 & 0.9091 & 1.0000 & -1 \\
500052565 & 1.0000 & 1.0000 & 1.0000 & 0 \\
500065284 & 1.0000 & 1.0000 & 1.0000 & 0 \\
500007914 & 0.8182 & 1.0000 & 0.8182 & 2 \\
500065667 & 0.7273 & 0.8182 & 0.9091 & -1
\end{tabular}




\begin{tabular}{ccccc}
500065658 & 0.7273 & 0.8182 & 0.9091 & -1 \\
500065660 & 0.6364 & 0.7273 & 0.9091 & -2 \\
500071568 & 1.0000 & 1.0000 & 1.0000 & 0 \\
500020615 & 0.5455 & 0.6364 & 0.9091 & -3 \\
500071563 & 1.0000 & 1.0000 & 1.0000 & 0 \\
500074855 & 0.8182 & 0.8182 & 1.0000 & -2 \\
500029272 & 0.6364 & 0.7273 & 0.9091 & -3 \\
500069479 & 0.3636 & 0.4545 & 0.9091 & -5 \\
500032684 & 0.8182 & 0.9091 & 0.9091 & 0 \\
500007425 & 0.9091 & 0.9091 & 1.0000 & -1 \\
Average & 0.8364 & 0.8818 & 0.9545 & -1 \\
\hline
\end{tabular}

Table 2 Forecasting Accuracy of 20 materials

\begin{tabular}{|c|c|c|c|c|c|}
\hline Country code & error & $\begin{array}{l}\text { Method in } \\
\text { this paper }\end{array}$ & Croston & Trend-monthly & ES \\
\hline \multirow[t]{3}{*}{500031955} & MAE & 0.1364 & 0.1447 & 0.1879 & 0.1429 \\
\hline & RMSE & 0.4677 & 0.4861 & 0.4848 & 0.5524 \\
\hline & MASE & 0.8048 & 0.8535 & 1.0266 & 0.9857 \\
\hline \multirow[t]{3}{*}{500067198} & MAE & 4.9361 & 6.4258 & 6.1936 & 5.2717 \\
\hline & RMSE & 7.8157 & 8.9960 & 8.9071 & 9.8051 \\
\hline & MASE & 0.8189 & 0.9526 & 0.9182 & 0.9162 \\
\hline \multirow[t]{3}{*}{500037055} & MAE & 0.0195 & 0.0370 & 0.0335 & 0.0286 \\
\hline & RMSE & 0.1236 & 0.1356 & 0.1254 & 0.1532 \\
\hline & MASE & 0.6367 & 1.0915 & 0.9892 & 0.9857 \\
\hline \multirow[t]{3}{*}{500072175} & MAE & 0.0757 & 0.1310 & 0.1333 & 0.1137 \\
\hline & RMSE & 0.3031 & 0.3072 & 0.4830 & 0.3747 \\
\hline & MASE & 0.6285 & 0.9661 & 0.9833 & 0.9810 \\
\hline \multirow[t]{3}{*}{500026753} & MAE & 0.0012 & 0.0040 & 0.0023 & 0.0076 \\
\hline & RMSE & 0.0089 & 0.0090 & 0.0090 & 0.0317 \\
\hline & MASE & 0.1578 & 0.4917 & 0.9803 & 0.9857 \\
\hline \multirow[t]{3}{*}{500065263} & MAE & 0.0001 & 0.0002 & 0.0003 & 0.0004 \\
\hline & RMSE & 0.0007 & 0.0008 & 0.0007 & 0.0012 \\
\hline & MASE & 0.3626 & 0.4659 & 0.9671 & 0.9714 \\
\hline \multirow[t]{3}{*}{500052565} & MAE & 0.0025 & 0.0048 & 0.0044 & 0.0037 \\
\hline & RMSE & 0.0161 & 0.0177 & 0.0163 & 0.0199 \\
\hline & MASE & 0.6367 & 1.0968 & 0.9892 & 0.9857 \\
\hline \multirow[t]{3}{*}{500065284} & MAE & 0.0003 & 0.0004 & 0.0006 & 0.0005 \\
\hline & RMSE & 0.0022 & 0.0023 & 0.0023 & 0.0028 \\
\hline & MASE & 0.4917 & 0.6367 & 0.9892 & 0.9857 \\
\hline \multirow[t]{3}{*}{500007914} & MAE & 2.1845 & 2.6390 & 2.5867 & 2.6719 \\
\hline & RMSE & 3.7477 & 4.2751 & 4.1550 & 4.9387 \\
\hline & MASE & 0.6864 & 0.8948 & 0.8771 & 0.9311 \\
\hline \multirow[t]{3}{*}{500065667} & MAE & 0.8999 & 1.1333 & 1.0378 & 0.9918 \\
\hline & RMSE & 1.2947 & 1.3880 & 1.4548 & 1.4443 \\
\hline & MASE & 0.7920 & 0.9980 & 0.9139 & 0.9638 \\
\hline \multirow[t]{3}{*}{500065658} & MAE & 2.0290 & 2.7560 & 2.8186 & 2.5399 \\
\hline & RMSE & 2.9983 & 3.5566 & 3.5945 & 3.6919 \\
\hline & MASE & 0.9396 & 1.1783 & 1.2050 & 1.1516 \\
\hline \multirow[t]{3}{*}{500065660} & MAE & 1.2326 & 1.5010 & 1.4278 & 1.4547 \\
\hline & RMSE & 1.7955 & 2.0304 & 1.9066 & 2.1019 \\
\hline & MASE & 0.7430 & 0.9130 & 0.8685 & 0.9469 \\
\hline \multirow[t]{3}{*}{500071568} & MAE & 0.0689 & 0.1298 & 0.1329 & 0.1143 \\
\hline & RMSE & 0.5070 & 0.5232 & 0.5116 & 0.6129 \\
\hline & MASE & 0.5974 & 0.9575 & 0.9803 & 0.9857 \\
\hline
\end{tabular}




\begin{tabular}{lccccc}
500020615 & MAE & 15.9885 & 17.1784 & 19.9635 & 17,1360 \\
500071563 & RMSE & 28.6579 & 29.2930 & 32.3716 & 31.9071 \\
& MASE & 0.8125 & 0.8730 & 0.9288 & 0.9621 \\
500074855 & MAE & 0.0590 & 0.0609 & 0.1801 & 0.0857 \\
& RMSE & 0.3724 & 0.3880 & 0.3917 & 0.4596 \\
500029272 & MASE & 0.5802 & 0.6214 & 1.7711 & 0.9857 \\
& MAE & 0.0590 & 0.0609 & 0.1801 & 0.0857 \\
500069479 & RMSE & 0.3724 & 0.3880 & 0.3917 & 0.4596 \\
& MASE & 0.5802 & 0.6214 & 1.7711 & 0.9857 \\
& MAE & 1.5912 & 2.1627 & 2.1817 & 2.1303 \\
500032684 & MASE & 2.4745 & 2.8674 & 2.8275 & 3.1953 \\
& MAE & 0.6316 & 0.9594 & 0.9678 & 0.9734 \\
500007425 & RMSE & 4.2666 & 4.2677 & 4.8952 & 3.4323 \\
& MASE & 0.9504 & 5.6296 & 6.2870 & 5.9882 \\
& MAE & 1.5872 & 1.9047 & 1.2557 & 1.1166 \\
& RMSE & 2.8295 & 3.1383 & 1.8746 & 1.8764 \\
& MASE & 0.7865 & 0.8599 & 3.0920 & 3.5917 \\
& MAE & 1.0706 & 1.2417 & 0.8778 & 0.9662 \\
5 & RMSE & 3.1483 & 3.4101 & 1.5787 & 1.2540 \\
& MASE & 0.6064 & 0.9392 & 3.4103 & 4.1944 \\
& & & & 1.1942 & 0.9614 \\
\hline
\end{tabular}

As there is no existing predicting method aiming for demand occurrence (SEC method based on time series analysis, Croston method together with improvement have forecasted number in every given time but they can only provide average demand prediction for a given period; Bootstrap method that roots in statistics could cater for offering the probability of demand occurrence in the next given time while it still cannot give exact values), this article only provided the accuracy of MAP mentioned in the previous paragraph. From the predicted result of actual historical data, this method has a high prediction accuracy rate; from the twenty sample data collected, it has an $83.64 \%$ of aiming rate; seven sample data even has a $100 \%$ aiming rate. In conclusion, this is a probable prediction method. However, as shown in Table 1, in the prediction of the possibility of actual material demand occurrences, the accuracy of the prediction of happening is lower than that of not happening, and the data of Miss Demands also shew, the possibility of under stating is way higher than overstating its demand. Therefore, first of all, improvement should be made, for example, when using MAP to forecast time points of demand arrivals, it is of no certain need to recognize there will be demand appearing in $\mathrm{t}$ period only if $p_{1} \geq p_{0}$ ,varying $p_{r} 0 \leq p_{r} \leq 1$, to adapt to different situations of materials; when $p_{1} \geq p_{r}$, recognize that there will be demand occurrence and further prediction is needed, otherwise no demand should be recognized and there is no need to further prediction, in this way prediction probability could be lifted to a higher level; secondly, in actual practices, safety stock are supposed to be used to guarantee timely supply of electrical materials. 
Furthermore, it is apparent that according to the experiment results of Table 2, method designed in this essay is more superior compared to results of other methods in 95 percent tests. In addition, the designed method shows less error values on all selected error indicator in 95 percent tests. From the aspect of the most discriminant indicator, MASE, the average value of the designed method is 0.6418 that of exponential smoothing is 0.9835 , and the average value of Croston, season-tendency prediction is separately 0.8707 and 1.910. Seen from the comparisons of average number of MASE, the value of designed approach is much less of others; and the experiment data of selected material is 32-95 percent of Croston, 8-90 percent of seasontendency prediction and 16-90 percent of exponential smoothing method separately. So, the empirical results prove that MAP, method designed in this paper is a relatively effective approach as for predicting data with intermittent characteristics.

\section{Conclusion}

This article suggested a digital decomposition based demand intermittent prediction method. This splitting prediction method reduces the difficulty, gives contributes into discovering hidden pattern and provides a new way of thinking and method for demand prediction of electrical materials. There is also empirical evidence that this way is a feasible and practical for inventory management, with high accuracy level. Nevertheless, because the probability of losing demands numbers is larger than overstating them, when using MAP approach to forecast demand occurrence point, it is allowed soften the term that probability of demand occurrence to lower the risk of failing to declare demand. Apart from that, from the perspective of satisfying needs, stepping further toward paying more attention to suppliers and electrical materials offering is one significant study direction for this paper.

\section{Acknowledgements}

The research described in this paper has been funded by the National Natural Science Foundation of China (Grant No. 71302053)

\section{References :}

Silver E A, Operations research in inventory management: A review and critique [J]. Operations Research, 1981, 29: 628-645.

ZS Hua, B Zhang, J Yang and DS Tan, A new approach of forecasting intermittent demand for spare parts inventories in the process industries [J]. Journal of the Operational Research Society, 2007, 58, 52-61.

Willemain T R, Smart C N, Schwarz H F, A new approach to forecasting 
intermittent demand for service parts inventories [J]. International Journal of Forecasting, 2004, 20: 375-387.

Ghobbar A A, Friend C H, Evaluation of forecasting methods for intermittent parts demand in the field of aviation: a predictive model [J]. Computers \& Operations Research, 2003, 30:2097-2114.

Rustenburg W D, van Houtum GJ, Zijm WHM, Spare parts management at complex technology-based organizations: An agenda for research [J]. International Journal of Production Economics, 2001, 71:1772193.

Gardner E S, Exponential smoothing: the state of the art [J]. Journal of Forecasting, 1985, 4: 1-28.

Croston J D, Forecasting and stock control for intermittent demands [J]. Operational Research Quarterly, 1972, 23 (3): 289-303.

Gamero M J, García J M, Reyes A M, Bootstrapping statistical functions [J]. Statistics \& Probability Letters, 1998, 39 (3): 229-236.

Efron B, Bootstrap methods: another look at the jackknife [J]. Annals of Statistics, 1979, 7: 1-26.

Snyder R, Forecasting sales of slow and fast moving inventories [J]. European Journal of Operational Research, 2002, 140: 684-699.

Leven E, Segerstedt A, Inventory control with a modified croston procedure and Erlang distribution [J]. International Journal of Production Economics, 2004, 90:361-367.

Grange F, Challenges in modeling demand for inventory optimization of slow-moving items [C]. Proceedings of the $30^{\text {th }}$ conference on Winter simulation IEEE Computer Society Press, 1998, 1211-1218.

Silver E A, Bayesian determination of the reorder point of a slow moving item [J]. Operations Research, 1965, 13 (5): 989-997. 